\title{
Four-Wave-Mixing Gain and All-optical Signal Processing in Silicon Nanowires
}

\author{
Bart Kuyken $^{1 *}$, Xiaoping Liu ${ }^{2}$, Stéphane Clemmen ${ }^{3}$, Shankar Kumar Selvaraja ${ }^{1}$, Wim Bogaerts ${ }^{1}$, Dries Van Thourhout ${ }^{1}$, \\ Richard M. Osgood, Jr. ${ }^{2}$, Philippe Emplit ${ }^{3}$, Serge Massar ${ }^{3}$, Yurii A. Vlasov ${ }^{4}$, \\ William M. J. Green ${ }^{4}$, Günther Roelkens ${ }^{1}$, Roel Baets ${ }^{1}$ \\ ${ }^{1}$ Photonics Research Group, Department of Information Technology, Ghent University - imec, Ghent, Belgium \\ ${ }^{2}$ Department of Electrical Engineering, Columbia University, 1300 S. W. Mudd, 500 W. 120th St., New York, NY 10027, USA \\ ${ }^{3}$ Laboratoire d'Information Quantique (LIQ), Université Libre de Bruxelles (U. L. B.), \\ ${ }^{4}$ IBM T. J. Watson Research Center, Yorktown Heights, NY 10598, USA \\ *Bart.Kuyken@intec.ugent.be
}

\begin{abstract}
Silicon nanowires have an immense potential for nonlinear optical functions. Recent work on four-wave-mixing gain in hydrogenated amorphous silicon wires at telecom wavelengths and in crystalline silicon wires in the $2 \mu \mathrm{m}$ wavelength range is reviewed.

OCIS codes: (190.4380) Four wave mixing; (130.4310) Nonlinear integrated optics
\end{abstract}

\section{Introduction}

All-optical processing allows increasing the capacity of future telecommunication networks, while at the same time drastically reducing the power consumption of those networks. The high nonlinear index of crystalline silicon, combined with the high confinement in silicon nanowires due to its high linear index have made the silicon-oninsulator platform an excellent platform to do low-power nonlinear optics and all-optical signal processing. Alloptical functions such as wavelength conversion [1], signal regeneration [2] and parametric gain [3] have been demonstrated. However, at telecom wavelengths crystalline silicon suffers, as a result of the two-photon absorption process (TPA), from extensive nonlinear absorption. This translates in a poor Figure of Merit (FOM) for crystalline silicon, defined as the ratio of the nonlinear refraction to the nonlinear absorption. This nonlinear absorption has limited the performance of these all-optical functions enormously; for example, the parametric gain achieved in crystalline silicon at telecom wavelengths is only a few $\mathrm{dB}[3]$.

A first approach to overcome the TPA problem is to step away from the telecommunication wavelength and work at wavelengths where TPA is absent, as explained in the last part of this paper. Another approach to suppress the nonlinear absorption is the use of another nonlinear core material. Here we present hydrogenated amorphous silicon (a-Si:H) with a reduced nonlinear absorption and higher nonlinear index as a novel nonlinear material for the telecom wavelength range. We demonstrate a FOM four times higher than its equivalent in crystalline silicon.

\section{Hydrogenated amorphous silicon}

Photonic wires fabricated froma-Si:H are considered to be a good alternative for the standard crystalline silicon-oninsulator photonic wires. The refractive index of a-Si:H is similar to (actually slightly slightly higher than) its crystalline counterpart, resulting in highly integrated photonic circuits. Moreover, a-Si:H layers can be deposited at low temperatures and thus be back-end deposited on a finished CMOS wafer. Several integrated photonic components based on the amorphous silicon platform, including high Q resonators, Mach-Zehnder interferometers and low-loss $(3.6 \mathrm{~dB} / \mathrm{cm})$ single mode photonic wires have been demonstrated [4].

Highly nonlinear a-Si:H photonic wires were defined by depositing a $220 \mathrm{~nm}$ a-Si:H layer on top of a $1950 \mathrm{~nm}$ polished silicon dioxide layer on a silicon substrate in a CMOS pilot line. The photonic wires were patterned using wafer-scale CMOS fabrication technology. The obtained $500 \mathrm{~nm}$ wide photonic wires have no upper cladding layer. The high confinement obtained by the high lateral and vertical index contrast not only enhances the nonlinear response of the photonic wire, it also allows for dispersion engineering of the photonic wire. The wire was engineered to have an anamolous dispersion of $\beta_{2}=-2.0 \mathrm{ps}^{2} / \mathrm{m}$. The nonlinear absorption and nonlinear response of the wire were estimated by measuring the nonlinear transmission of the waveguide and by comparing the modulation fringes of the output spectrum when a picoseconds pulse train is injected in the photonic wire, respectively [5]. This procedure revealed a record nonlinear parameter of 770/Wm for the photonic wire and a FOM of 2.1. The modest nonlinear absorption and high nonlinear response make this material a good candidate for alloptical signal processing.

The high nonlinear response of the photonic wires was demonstrated in a pump-probe experiment [6]. For this experiment, an intense pump pulse was combined with a weak signal pulse in a $1.1 \mathrm{~cm}$ long photonic wire (500nm by $220 \mathrm{~nm}$ ). An on/off gain of $26.5 \mathrm{~dB}$ was obtained, using 4 ps pump pulses with a coupled peak power of $5.3 \mathrm{~W}$, as shown in figure 1 . This is an improvement of more than $20 \mathrm{~dB}$ over the result obtained in crystalline silicon at 
telecom wavelengths. Moreover, the bandwidth of the nonlinear amplifier spans more than $100 \mathrm{~nm}$ (versus $15 \mathrm{~nm}$ in crystalline silicon at telecom wavelengths).
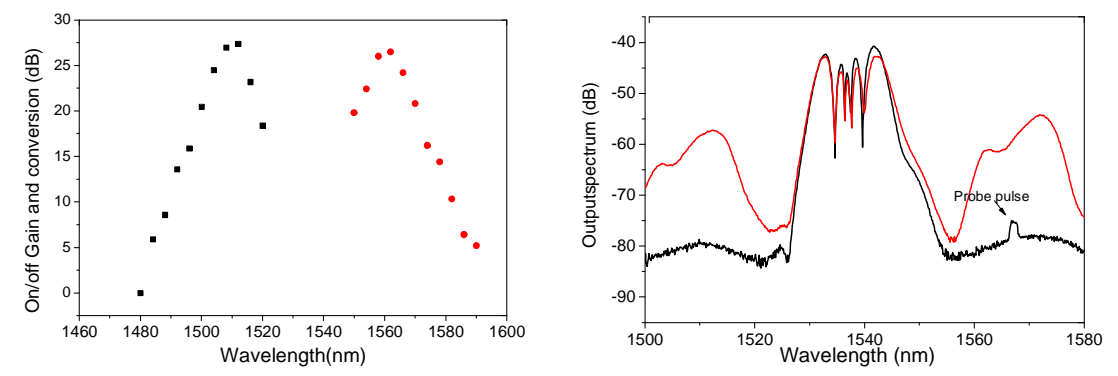

Fig. 1: The on/off gain of a weak probe pulse due to a high power $(5.3 \mathrm{~W})$ pump pulse at $1530 \mathrm{~nm}$ in a $1.1 \mathrm{~cm}$ long a-Si:H photonic wire is shown on the left. The gain is shown with red dots and the conversion efficiency of the signals is marked with black squares. On the right a typical output spectrum is shown, the black curve shows the output spectrum when the pump and probe pulse are not synchronized in time. The black curve shows the output spectrum when the pulses are synchronized in time.

The good nonlinear properties of the hydrogenated amorphous silicon are believed to result from the wider bandgap of the material compared to crystalline silicon. Measuring the bandgap by spectroscopic ellipsometry revealed a value of $1.61 \mathrm{eV}$, such that the energy of the telecom wavelength photons is close to half the band gap of the material. However, due to the amorphous structure of the material, the bandgap is not rigorously defined, which gives rise to Urbach band tails, such that two-photon absorption is not completely eliminated. Given the long free carrier lifetime, this can result in patterning effects in all-optical signal processing, if no care is taken to sweep out the carriers, e.g. using a lateral p-i-n junction. Also, the carriers created by the two photon process are believed to be the cause of the degradation of the a-Si:H material, which is observed when the material is exposed to high power pulses. In this process - called the Staebler-Wronski effect [7], well known from amorphous silicon solar cells - the energy released by the recombination of an electron-hole pair breaks weak silicon-silicon bonds and thereby creates defect states. Further optimization of the material system is underway to improve its stability.

\section{Nonlinear optics in crystalline silicon at longer wavelengths}

By moving to longer wavelengths $(\lambda \approx 2.2 \mu \mathrm{m})$, where the photon energy is close to the half band gap energy of crystalline silicon, the two-photon absorption can be surpressed enormously and nonlinear interactions become very efficient. By pumping properly designed waveguides we have recently demonstrated a supercontinuum spanning from the telecommunication wavelength into the mid-infrared [8]. By proper waveguide dispersion engineering a link to the telecom wavelength window can however be kept through wavelength conversion. To achieve this, we use higher orders of dispersion to achieve the phase matching condition between signal (in the telecom wavelength range), pump ( $2 \mu \mathrm{m}$ wavelength range) and idler (short-wave infrared wavelength range). Usually parametric gain is achieved in a band around the pump wavelength where the phasematching condition

$$
\beta_{2}(\Delta \omega)^{2}=2 \gamma P
$$

is satisified. In this equation $\Delta \omega$ is the detuning from the pump, $\gamma$ the nonlinear parameter, $P$ the coupled pump power and $\beta_{2}$ the group velocity dispersion at the pump. Indeed we have demonstrated that by pumping a waveguide close to its zero dispersion wavelength an enormous parametric gain can be obtained [9] in a $2 \mathrm{~cm}$ photonic wire. By pumping these wires with bright pump pulses (repetition rate $76 \mathrm{MHz}$, pulse duration $2 \mathrm{ps}$ at $2173 \mathrm{~nm}$ ) and using a $\mathrm{CW}$ signal as a probe a gain bandwidth of more than $580 \mathrm{~nm}$ was demonstrated as well as a maximum amplification of more than $50 \mathrm{~dB}$. However, when the signal detuning from the pump gets large, higher order dispersion terms have to be taken in account. It can be shown that in this case the phasematching relation is altered to

$$
\beta_{2}(\Delta \omega)^{2}+\frac{1}{12} \beta_{4}(\Delta \omega)^{4}=2 \not P
$$

Here $\beta_{4}$ is the fourth order dispersion. This equation has a solution close to the pump where the fourth order dispersion can be neglected and where the equation reduces to equation (1). However the relation also has a solution far away from the pump. A $900 \mathrm{~nm}$ wide crystalline silicon photonic wire, optimized to satisfy this relation [10] for telecom wavelengths using a pump around $1946 \mathrm{~nm}$, where the nonlinear index of crystalline silicon peaks, was fabricated. These wires were also processed in a CMOS pilot line on $200 \mathrm{~mm}$ SOI wafers, consisting of a $220 \mathrm{~nm}$ 
silicon waveguide layer on a $2 \mu \mathrm{m}$ buried oxide layer. The net on-chip conversion of a signal from the U-band to the short-waved infrared and vice versa was demonstrated. This is shown in figure 2, for a $2 \mathrm{~cm}$ long photonic wire. The lower conversion from the telecom wavelength range to the short-wave infrared is believed to be the result of cross two-photon absorption. This conversion scheme opens a new way for optical signal processing on chip, in which signals at telecom wavelengths could be converted to the short-wave infrared to do highly efficient all-optical signal processing, since TPA is absent and the nonlinear interactions are efficient. Afterwards this SWIR signal can be converted back to the telecom window.
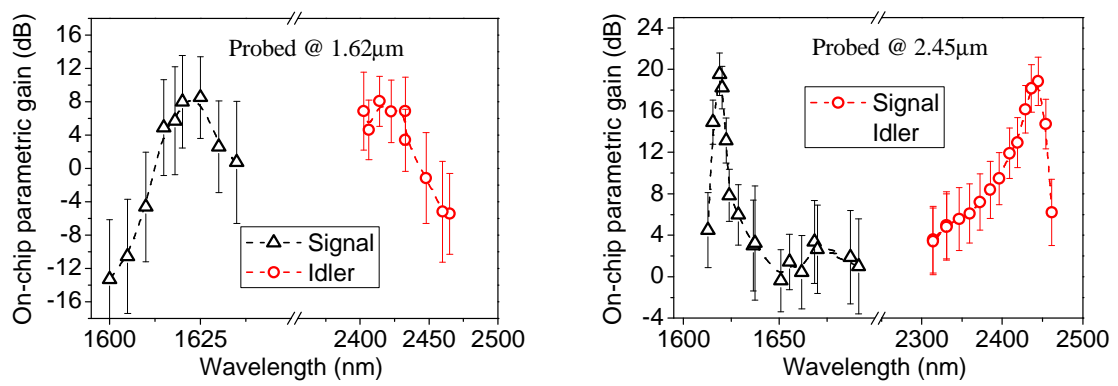

Fig. 2 The net-on chip conversion efficiency and gain for a signal in the telecom wavelength range which is converted to the short-wave infrared on the left and the conversion from the short-wave infrared to the telecom band on the right. These results were obtained at a coupled peak power of $37.3 \mathrm{~W}$ for a pump (rep rate $76 \mathrm{MHz}$, pulse duration $2 \mathrm{ps)}$ at $1946 \mathrm{~nm}$.

\section{Conclusions}

In summary, we have demonstrated that hydrogenated amorphous silicon has a large potential as a new nonlinear material at telecom wavelengths due to its wider band gap and larger nonlinear index. However, further engineering of the material is necessary to counteract the Staebler-Wrosnki effect. It is also shown that the conventional siliconon-insulator platform is an ideal candidate for nonlinear optics in the short-wave infrared. By proper engineering of silicon photonic wires the benefits of working at these wavelengths can be used for all-optical processing of telecom signals.

\section{References}

[1] Rong, H et al. "High efficiency wavelength conversion of $10 \mathrm{~Gb} / \mathrm{s}$ data in silicon waveguides." Opt. Express 14, 1182-1188 (2006).

[2] Salem, R. et al. "Signal regeneration using low-power four-wave mixing on silicon chip". Nature Photonics 2, 35-38 (2008).

[3] Foster, M. A. et al. "Broad-band optical parametric gain on a silicon photonic chip". Nature 441, 960-963 (2006).

[4] Selvaraja, S. et al. "Low-Loss Amorphous Silicon-On-Insulator Technology for Photonic Integrated Circuitry”,Optics Communications 9 , p. 1767-1770 (2009)

[5] Kuyken, B. et al. "Self phase modulation in highly nonlinear hydrogenated amorphous silicon “,Photonics Society Annual Meeting, Paper WS 3, United States, (2010)

[6] Kuyken, B. et al. "On-chip parametric amplification with $26.5 \mathrm{~dB}$ gain at telecommunication wavelengths using CMOS-compatible hydrogenated amorphous silicon waveguides", Optics Letters 36, p.552-554 (2011)

[7] Staebler, D. L. et al. "Optically induced conductivity changes in discharge-produced hydrogenated amorphous silicon". J. Appl. Physics. 51 (1980).

[8] Kuyken, B. et al. "Generation of a telecom-to-mid-infrared spanning supercontinuum using silicon-on-insulator wire waveguides", Conference on Laser and Electro-Optics (CLEO), Paper CTuS2, United States (2011)

[9] Liu, X. et al. "Mid-infrared broadband modulation instability and 50dB Raman assisted parametric gain in silicon photonic wires", Conference on Laser and Electro-Optics, Paper CTuS1 United States (2011)

[10]Kuyken, B. et al. "Frequency conversion of mid-infrared optical signals into the telecom band using nonlinear silicon nanophotonic wires", Optical Fiber Conference, Paper OThU4, United States, (2011) 